\section{Genome Assembly and Annotation of Botryosphaeria dothidea sdau11-99, a Latent Pathogen of Apple Fruit Ring Rot in China}

\author{
Chengming Yu, ${ }^{1}$ Yufei Diao, ${ }^{1}$ Quan Lu, ${ }^{2}$ Jiaping Zhao, ${ }^{3}$ Shengnan Cui, ${ }^{1}$ Cheng Peng, ${ }^{1}$ \\ Bangling He, ${ }^{1}$ Yuancun Liang, ${ }^{1}$ and Huixiang Liu ${ }^{1, \dagger}$ \\ ${ }^{1}$ Shandong Research Center for Forestry Harmful Biological Control Engineering and Technology, \\ College of Plant Protection, Shandong Agricultural University, Tai'an 271018, China \\ ${ }^{2}$ Research Institute of Forest Ecology, Environment and Protection, Chinese Academy of Forestry, \\ Beijing 100091, China \\ ${ }^{3}$ Institute of Forestry New Technology, Chinese Academy of Forestry, Beijing 100091, China
}

\section{Abstract}

Botryosphaeria dothidea is a latent and important fungal pathogen on a wide range of woody plants. Fruit ring rot caused by $B$. dothidea is a major disease in China on apple. This study establishes a high-quality, nearly complete, and well-annotated genome sequence of $B$. dothidea strain sdau11-99. The findings of this research provide a reference genome resource for further research on the apple fruit ring rot pathogen on apple and other hosts.

\section{Genome Announcement}

Botryosphaeria dothidea is one of the most important broad-host-range pathogens (Chen et al. 2016; Dissanayake 2016; Liu et al. 2009; Liu et al. 2018; Marsberg et al. 2017; Wang et al. 2018; Zhang et al. 2013), which causes woody plant fruit rot, stem canker, and twig dieback diseases. Apple fruit ring rot caused by $B$. dothidea is a typical fruit disease that results in huge economic losses in apple production regions. Strain sdau11-99 of $B$. dothidea was isolated from infected cultivar Fuji apple fruit in an orchard, unmanaged for many years, in Rongcheng of Shandong Province of China in 2010. On potato glucose agar medium, the colonies were initially gray, then darker, finally turning gray/black. The aerial hyphae were well developed. The virulence of the strain was higher than other strains that were isolated from other regions in China. Upon microscopic observation, fruiting bodies were spherical, and gray to black. Pycnidia were single or aggregated, with multiple chambers. Conidia were spindle or ovoid, colorless, and hyaline with a size of 16.87 to $24.39 \times 2.40$ to $6.26 \mu \mathrm{m}$, and the length/width ratio of conidia was 3.9 to 7.03. Based on the morphological features and multiple locus sequencing typing using ribosomal DNA-internal transcribed spacer, $\beta$-tubulin, elongation factor- $1 \alpha$, rpb2, large subunit, and small subunit, strain sdau11-99 was identified as B. dothidea (unpublished data).

The genome of $B$. dothidea sdau11-99 was sequenced using PacBio Sequel II System (PacBio, Menlo Park, CA, U.S.A.) and Illumina NovaSeq 6000 System (Illumina, San Diego, CA, U.S.A.). In total, 1,101,769 PacBio long reads and 19,848,356 Illumina short reads were obtained, representing genomic coverage of $153 \times$ and $114.5 \times$, respectively. De novo genome assembly was conducted with high-quality PacBio long reads using Falcon v1.2.6 (Chin et al. 2016) and Canu v1.8 software (Koren et al. 2017) with the default parameters, resulting in 86 contiguous segments (contigs) of $48,010,522$ bp with $N_{50}$ values of $1,404,133$ bp and 140

${ }^{\dagger}$ Corresponding author: H. Liu; hxliu722@126.com

The author(s) declare no conflict of interest.

Accepted for publication 28 November 2020 .

\section{Funding}

Support was provided by the National Key Research and Development Plan of China (grant number 2017YFD0600102-7) and National Natural Science Foundation of China (grant number 31770684).

\section{Keywords}

fungi, pathogen diversity, trees 
Table 1. Whole-genome comparison analysis of Botryosphaeria dothidea sdau11-99 and other $B$. dothidea assemblies

\begin{tabular}{lccccc} 
Parameters & sdau11-99 & LW030101 & CBS_115476 & PG45 & LW-Hubei \\
Genome assembly length (bp) & $51,758,482$ & $47,389,336$ & $43,500,615$ & $44,169,592$ & $46,346,934$ \\
Number of scaffolds & 88 & 1,134 & 1,711 & 420 & 49 \\
GC contents (\%) & 52.93 & 53.10 & 52.30 & 54.60 & 54.30 \\
$\mathrm{~N}_{50}$ (bp) & $4,039,455$ & 287,735 & 86,212 & 352,475 & $3,265,504$ \\
BUSCO completeness (\%) & 97.3 & 98.90 & 98.80 & 98.90 & 98.5 \\
Average nucleotide identity (\%) & $\ldots$ & 99.97 & 96.76 & 96.72 & 96.74 \\
Reference & This work & Liu et al. 2016 & Marsberg et al. 2017 & Wang et al. 2018 & GenBank database \\
\hline
\end{tabular}

contigs of 50,501,013 bp with $\mathrm{N}_{50}$ values of $1,799,068 \mathrm{bp}$, respectively. The contigs were corrected and optimized by self-alignment using the Arrow algorithm in Genomic Consensus v2.3.2 package (https://github.com/PacificBiosciences/GenomicConsensus/releases/tag/ 2.3.2) with the default parameters. The two assemblies were merged by Quickmerge v0.3 software (Chakraborty et al. 2016) to produce a contiguous assembly. The merged genome assembly was further corrected with Illumina short reads using the Pilon algorithm in the Genomic Consensus package with the default parameters. The final genome assembly included $51,758,482$ bp containing 88 scaffolds with $\mathrm{N}_{50}$ values of $4,039,455 \mathrm{bp}$ and $\mathrm{GC}$ content of $52.93 \%$. To evaluate the quality of the genome assembly, the Illumina short reads and PacBio long reads were mapped to the genome assembly using BWA v0.7.17 ( $\mathrm{Li}$ and Durbin 2009) and Blasr v5.1 (Chaisson and Tesler 2012) software, which showed alignment with genome mapping ratios of 91.23 and $95.86 \%$, respectively. Meanwhile, the completeness of genome assembly was assessed by BUSCO v4.0.0 (Simão et al. 2015) against 1,315 ascomycota single-copy Benchmarking Universal Single-Copy Orthologs (BUSCOs), which showed that $97.3 \%$ of the gene sets in the genome assembly consisted of whole, single-copy BUSCOs. These results indicated that the genome was well assembled, with a high completeness.

Repeat elements of the genome assembly were annotated by integrating homology alignments and de novo predictions (Jurka et al. 2005; Tarailo-Graovac and Chen 2009). In total, $7,712,198$ bp ( $14.83 \%$ of genome assembly) of repeat sequences were identified. These primarily included $3,498,517 \mathrm{bp}(6.73 \%)$ of long terminal repeat retrotransposons and $2,042,543 \mathrm{bp}(3.93 \%)$ of long interspersed nuclear elements. Based on the soft repeat masked genome assembly, the protein coding genes were predicted using ab initio gene predictions and homology-based methods. In total, 13,216 and 14,816 genes were identified by using AUGUSTUS v2.5.5 software (Hoff and Stanke 2019) and GeneMark-ES v4.38 software (Borodovsky and Lomsadze 2011), respectively. Using the homology-based gene predictor of Exonerate v2.4.7 (Slater and Birney 2005), 16,802 genes were identified in the genome assembly. All of the independent gene sets were merged as the final gene models using EVidenceModeler v1.1.1 software (Haas et al. 2008), resulting in a total of 14,118 gene models. In addition, in total, 60 ribosomal RNA genes and 402 transfer RNA (tRNA) genes were also identified in the genome assembly using the RNAmmer v1.2 (Lagesen et al. 2007) and tRNAscan-SE v2.0 software (Schattner et al. 2005). Among the gene models, about $71.86 \%$ of the gene models were successfully annotated using InterProScan v5.33-72.0 (Hunter et al. 2009). Furthermore, based on the gene family prediction methods previously reported (Choi et al. 2010, 2013, 2014; Nelson 2009), in total, 29 peroxidase, 311 cytochrome P450, 274 transcription factor, 317 plant cell wall degradation, and 1,820 secreted proteincoding genes were predicted in the genome assembly. In addition, genes encoding 287 lipases, 798 CAZymes, and 1,293 peptidases were predicted against the dbCAN (release 8.0), MEROPS (release 12.1), and LED (release 3.0) databases (Barrett et al. 2001; Fischer and Pleiss 2003; Zhang et al. 2018), respectively. Whole-genome comparison analyses by MUMmer v4.0 (Kurtz et al. 2004) with strain sdau11-99 and other strains of B. dothidea, including the CBS 115476, PG45, LW030101 (Liu et al. 2016; Marsberg et al. 2017; Wang et al. 2018), and LW-Hubei strains, showed that strain sdau11-99 shares 96.72 to $99.97 \%$ average nucleotide coverage with other strains, with the highest similarity to LW030101 (Table 1). The high similarities of the $B$. dothidea strains indicate that the pathogen is highly conserved and widely distributed.

In summary, a high-quality and nearly complete genomic sequence of $B$. dothidea strain sdau11-99 was established. The comprehensive analyses of the whole genomic sequence of 
B. dothidea will provide critical insights into understanding the mechanism of gene regulation, gene function, evolution characteristics, and control of disease. The whole genomic sequence of $B$. dothidea sdau11-99 is available in the NCBI GenBank database with the accession number of WWBZ00000000.

\section{Literature Cited}

Barrett, A. J., Rawlings, N. D., and Obrien, E. A. 2001. The MEROPS database as a protease information system. J. Struct. Biol. 134:95-102.

Borodovsky, M., and Lomsadze, A. 2011. Eukaryotic gene prediction using GeneMark.hmm-E and GeneMark-ES. Curr. Protoc. Bioinf. 35:4.6.1-4.6.10.

Chaisson, M., and Tesler, G. 2012. Mapping single molecule sequencing reads using basic local alignment with successive refinement (BLASR): Application and theory. BMC Bioinf. 13:238.

Chakraborty, M., Baldwin-Brown, J. G., Long, A. D., and Emerson, J. J. 2016. Contiguous and accurate de novo assembly of metazoan genomes with modest long read coverage. Nucleic Acids Res. 44:e147.

Chen, L., Wang, Q., Chen, H., Sun, G., Liu, H., and Wang, H. 2016. Agrobacterium tumefaciens-mediated transformation of Botryosphaeria dothidea. World J. Microbiol. Biotechnol. 32:106.

Chin, C.-S., Peluso, P., Sedlazeck, F. J., Nattestad, M., Concepcion, G. T., Clum, A., Dunn, C., O'Malley, R. C., Figueroa-Balderas, R., Morales-Cruz, A., Cramer, G. R., Delledonne, M., Luo, C., Ecker, J. R., Cantu, D., Rank, D. R., and Schatz, M. C. 2016. Phased diploid genome assembly with single-molecule real-time sequencing. Nat. Methods 13:1050-1054.

Choi, J., Detry, N., Kim, K., Asiegbu, F. O., Valkonen, J. P. T., and Lee, Y. 2014. fPoxDB: Fungal peroxidase database for comparative genomics. BMC Microbiol. 14:117.

Choi, J., Kim, K., Jeon, J., and Lee, Y. 2013. Fungal plant cell wall-degrading enzyme database: A platform for comparative and evolutionary genomics in fungi and oomycetes. BMC Genomics 14:S7.

Choi, J., Park, J., Kim, D., Jung, K., Kang, S., and Lee, Y. 2010. Fungal Secretome Database: Integrated platform for annotation of fungal secretomes. BMC Genomics 11:105.

Dissanayake, A. J. 2016. Botryosphaeriaceae: Current status of genera and species. Mycosphere 7:1001-1073.

Fischer, M., and Pleiss, J. 2003. The Lipase Engineering Database: A navigation and analysis tool for protein families. Nucleic Acids Res. 31:319-321.

Haas, B. J., Salzberg, S. L., Zhu, W., Pertea, M., Allen, J. E., Orvis, J., White, O., Buell, C. R., and Wortman, J. R. 2008. Automated eukaryotic gene structure annotation using EVidenceModeler and the Program to Assemble Spliced Alignments. Genome Biol. 9:R7.

Hoff, K. J., and Stanke, M. 2019. Predicting genes in single genomes with AUGUSTUS. Curr. Protoc. Bioinf. 65:e57.

Hunter, S., Apweiler, R., Attwood, T. K., Bairoch, A. M., Bateman, A., Binns, D., Bork, P., Das, U., Daugherty, L., and Duquenne, L. 2009. InterPro: The integrative protein signature database. Nucleic Acids Res. 37:D211-D215.

Jurka, J., Kapitonov, V. V., Pavlicek, A., Klonowski, P., Kohany, O., and Walichiewicz, J. 2005. Repbase Update, a database of eukaryotic repetitive elements. Cytogenet. Genome Res. 110:462-467.
Koren, S., Walenz, B. P., Berlin, K., Miller, J. R., Bergman, N. H., and Phillippy, A. M. 2017. Canu: Scalable and accurate long-read assembly via adaptive k-mer weighting and repeat separation. Genome Res. 27:722-736.

Kurtz, S., Phillippy, A. M., Delcher, A. L., Smoot, M. E., Shumway, M., Antonescu, C., and Salzberg, S. L. 2004. Versatile and open software for comparing large genomes. Genome Biol. 5:R12.

Lagesen, K., Hallin, P. F., Rodland, E. A., Staerfeldt, H., Rognes, T., and Ussery, D. W. 2007. RNAmmer: Consistent and rapid annotation of ribosomal RNA genes. Nucleic Acids Res. 35:3100-3108.

$\mathrm{Li}, \mathrm{H}$., and Durbin, R. 2009. Fast and accurate short read alignment with BurrowsWheeler transform. Bioinformatics 25:1754-1760.

Liu, H. X., Li, X. D., Zhu, X. P., and Liu, A. X. 2009. First report of pomegranate stem scab caused by Botryosphaeria dothidea in China. Plant Pathol. 58:400.

Liu, X., Liu, H. X., Han, X., Zhang, Y., Liang, Q., Li, S., and Yang, K. 2018. First report of Botryosphaeria dothidea causing fruit rot of yellowhorn (Xanthoceras sorbifolium) in China. Plant Dis. 102:1662.

Liu, Z., Lian, S., Li, B., Lu, H., Dong, X., and Wang, C. 2016. Draft genome sequence of Botryosphaeria dothidea, the pathogen of apple ring rot. Genome Announce. 4:e01142-16.

Marsberg, A., Kemler, M., Jami, F., Nagel, J. H., Postmasmidt, A., Naidoo, S., Wingfield, M. J., Crous, P. W., Spatafora, J. W., and Hesse, C. 2017. Botryosphaeria dothidea: A latent pathogen of global importance to woody plant health. Mol. Plant Pathol. 18:477-488.

Nelson, D. R. 2009. The cytochrome p450 homepage. Hum. Genomics 4:59-65.

Schattner, P., Brooks, A. N., and Lowe, T. M. 2005. The tRNAscan-SE, snoscan and snoGPS web servers for the detection of tRNAs and snoRNAs. Nucleic Acids Res. 33:W686-W689.

Simão, F. A., Waterhouse, R. M., Ioannidis, P., Kriventseva, E. V., and Zdobnov, E. M. 2015. BUSCO: Assessing genome assembly and annotation completeness with single copy orthologs. Bioinformatics 31:3210-3212.

Slater, G., and Birney, E. 2005. Automated generation of heuristics for biological sequence comparison. BMC Bioinf. 6:31.

Tarailo-Graovac, M., and Chen, N. 2009. Using RepeatMasker to identify repetitive elements in genomic sequences. Curr. Protoc. Bioinf. 25:4.10.1-4.10.14.

Wang, B., Liang, X., Gleason, M. L., Zhang, R., and Sun, G. 2018. Comparative genomics of Botryosphaeria dothidea and B. kuwatsukai, causal agents of apple ring rot, reveals both species expansion of pathogenicity-related genes and variations in virulence gene content during speciation. IMA Fungus 9:243-257.

Zhang, H., Yohe, T., Huang, L., Entwistle, S., Wu, P., Yang, Z., Busk, P. K., Xu, Y., and Yin, Y. 2018. dbCAN2: A meta server for automated carbohydrate-active enzyme annotation. Nucleic Acids Res. 46:W95-W101.

Zhang, Z., Deng, D., Qi, W., Fan, S., Cao, Y., Huang, J., and Liu, Z. 2013. Botryosphaeria dothidea, the causal agent of a new stem canker disease of Tatarian dogwood (Cornus alba) in China. Australas. Plant Pathol. 42:113-119. 